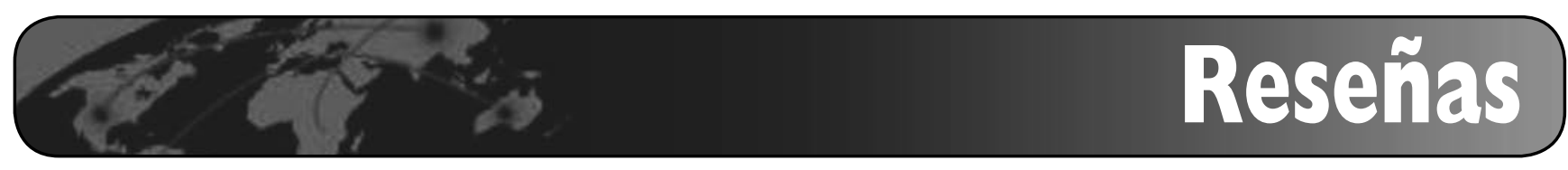

\title{
Pequeños y grandes desastres de la información (J. Cobarsí)
}

\author{
Por Sònia Aran-Ramspott
}

\begin{abstract}
Aran-Ramspott, Sònia. "Pequeños y grandes desastres de la información (J. Cobarsí)". Reseña de libro. En: El profesional de la información, 2007, mayo-junio, v. 16, n. 3, pp. 270-271.

DOI: 10.3145/epi.2007.may.13
\end{abstract}

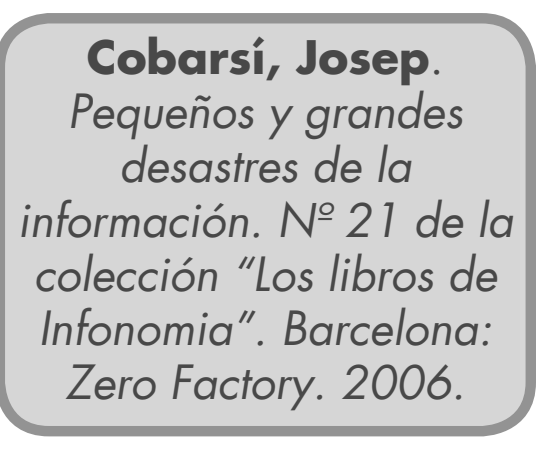

LAS CAUSAS Y CONSECUENCIAS DE LA MALA GESTIÓN DE LA INFORMACIÓN han merecido, generalmente, menos atención que los éxitos. En este libro, recopilación de casos verídicos, se emprende una exploración del lado oscuro de la información.

El autor ha ido analizando durante dos años en el boletín electrónico de Infonomía $i$-desastres una serie de casos muy variados de catástrofes, para rastrear las múltiples facetas de sus raíces y sus impactos, ya sea en nuestra vida diaria o en momentos clave para el destino de organizaciones o de países. En este volumen se combina una selección de esos artículos junto con otros hasta ahora inéditos. En palabras de Agustí Canals, autor del prólogo del libro y director de los Estudios de Ciencias de la Información y de la Comunicación de la UOC: "en la sociedad del conocimiento los peores desastres serán los desastres de información”.
En cuanto al enfoque del libro, destacar en primer lugar que las historias "mandan": es decir, los casos no son meros ejemplos para reforzar el discurso, sino que son el eje del texto y como tales se pretende tengan vida propia. El lector podrá construirse su propia interpretación, en base al texto de este libro y eventualmente de las fuentes de información citadas u otras que él mismo pueda encontrar. Y esa interpretación podrá resultar en mayor o menor medida coincidente con la propuesta, siempre a título provisional, por el autor.

En segundo lugar, los casos presentados se consideran "para todos los públicos". Es decir, el conjunto de historias comentadas se cree potencialmente significativo y sugerente para lectores de diversos perfiles profesionales relacionados con la información, tales como: diseñadores y desarrolladores de sistemas de información, responsables de la provisión de servicios de información, periodistas, directivos de empresas e instituciones, etc.

\section{Contenido}

Los casos expuestos se clasifican en 3 grandes apartados:

- la a menudo difícil relación entre información y toma de decisiones, ante peligros como la simplificación, o los sistemas verticales y no diversificados. Aquí se habla por ejemplo de los peligros del
Power Point como elemento potencialmente simplificador y su uso indebido ante la toma de decisiones de alto nivel; o de los días previos a la erupción volcánica en la Martinica en 1902, una catástrofe natural con trasfondo político;

- situaciones que tienen como hilo conductor una deficiente gestión de información que nace del concepto de considerar, en el fondo y ni que sea inconscientemente, a la información como un bien tangible, en vez de reconocerlo con todas las consecuencias como un intangible dotado de propiedades específicas. Aquí se comentan casos com el del llamado "niño balsero", la falsa noticia del "motorista congelado" o

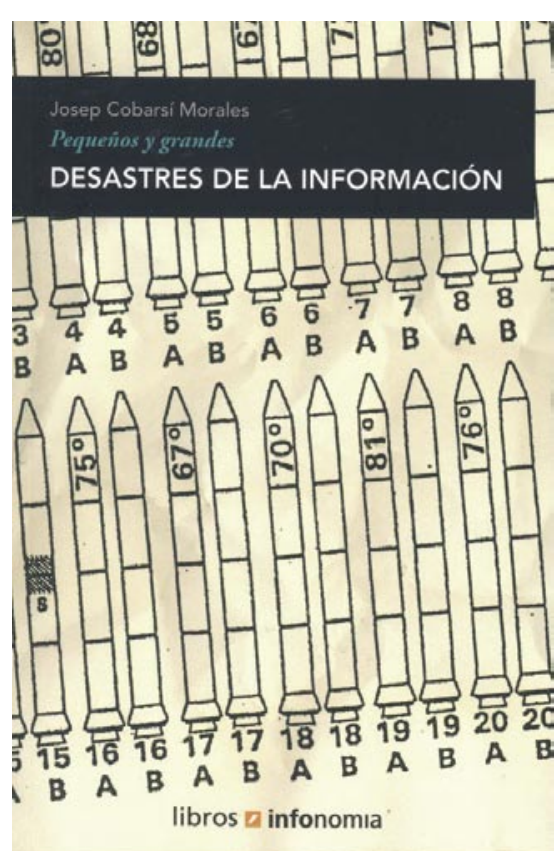


las dificultades en algún aplicativo informático de las olimpiadas de Atlanta 96;

- ciertas situaciones (que podríamos llamar "microdesastres" y "cuasidesastres) que parecen producirse asiduamente con patrones similares aquí y allá, sin tener necesariamente consecuencias catastróficas inmediatas. Aquí van, por ejemplo, la historia sobre la venta por teléfono o la de la "cola del correo electrónico".

El libro se cierra con la exposición sobre la "Verdadera historia de la Tabla Redonda", parábola de los problemas informacionales de las organizaciones de la sociedadred, en vertiginoso crecimiento en cuanto a dimensiones y complejidad.

El autor, Josep Cobarsí Morales, es profesor de los Estudios de Ciencias de la Información y de

\section{El coste de la desinformación}

El libro de Cobarsí trata en el fondo del gran dilema que se nos presenta cuando se trata de implementar servicios de información: ¿̇cuánto cuesta la información? ¿es rentable instalar un sistema de información? javamos a

tener un buen retorno de la inversión (ROI)?

Por desgracia para nuestra profesión es muy difícil calcular los beneficios producidos por un servicio de información, y mucho más todavía cuantificar los posibles desastres evitados gracias a su eficacia.

Cualquier problema, cualquier catástrofe pequeña o grande, cualquier cosa que haya ido mal se hubiera podido evitar teniendo la información adecuada, desde ataques terroristas a accidentes mecánicos, pasando por proyectos infructuosos y fracasos de todo tipo.

la Comunicación de la Universitat Oberta de Catalunya e investigador del Internet Interdisciplinary Institute de dicha universidad.
Sònia Aran-Ramspott, Centro de Cultura Contemporánea de Barcelona.

saran@cccb.org

Via Benedetto da Maiano 3

50014 Fiesole (Florencia)

Italia

Tel. +39055 50181

Fax +39055 5018201

info@casalini.it

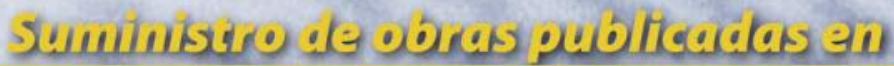

Strvicios Integrados para la biblioteca

Libros

Revistas

Approval Plan

Registros MARC

Bases de datos en línea razes 


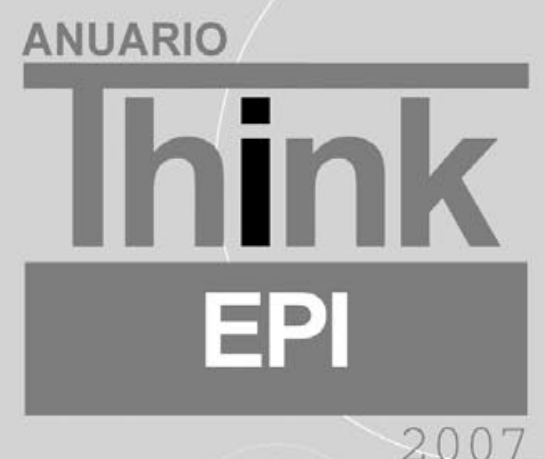

Análisis de tendencias en información y documentación

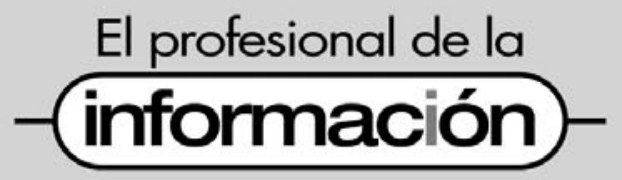

\section{SOLICITE UN EJEMPLAR DEL ANUARIO THINKEPI 2007}

Rellene este formulario (o su fotocopia) y envíelo:

- al apartado de correos 32.280 de 08080 Barcelona, o

- por correo-e a epi@sarenet.es

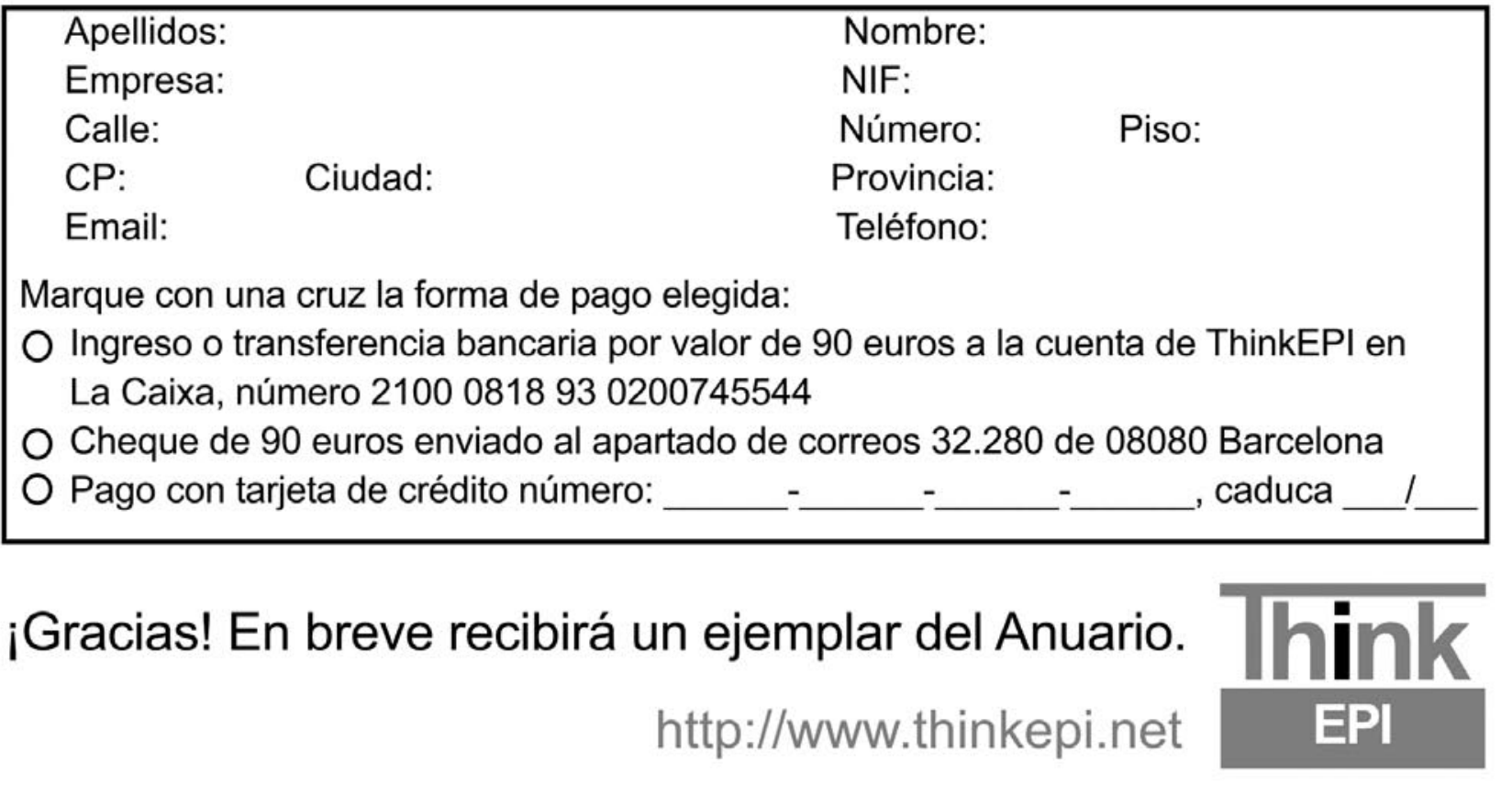

